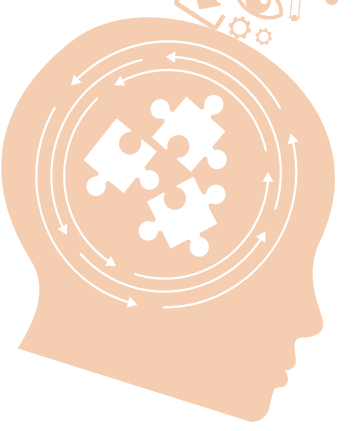

\title{
Gamificación y e-learning: estudio de un contexto universitario para la adecuación de su diseño
}

\begin{abstract}
Extracto:
La "gamificación», aplicada a la educación, puede afectar positivamente a la motivación e implicación del alumnado, si bien aún existen pocas investigaciones que respalden sus bondades y marquen pautas sobre cómo realizar este proceso. Este trabajo tiene como objetivo generar hipótesis dirigidas a facilitar el diseño de un futuro estudio piloto en el ámbito de la educación superior en modalidad e-learning. Para ello, se estudiaron factores relacionados con el alumnado y el profesorado del Máster de Educación y Nuevas Tecnologías de la Universidad a Distancia de Madrid (UDIMA). La metodología utilizada combina aspectos cualitativos y cuantitativos con una finalidad descriptiva y exploratoria. Para la obtención de datos se utilizaron dos cuestionarios on-line diferentes para el profesorado y el alumnado, incluyéndose en este último una traducción del test BrainHex. A partir de los datos y de la revisión de la literatura existente se generan conclusiones acerca de la aceptación del alumnado y del profesorado respecto a la introducción de la gamificación en la formación, sobre qué tipo de elementos de gamificación son más adecuados para su uso en el contexto de la UDIMA y a la motivación de qué aspectos o conductas deberían dirigirse para su éxito.
\end{abstract}

Fecha de entrada: 11-12-2014

Fecha de aceptación: 21-01-2015

Fecha de revisión: 08-04-2016

Palabras claves: gamificación, motivación, implicación, e-learning, modelo BrainHex, educación universitaria.

\footnotetext{
${ }^{1}$ M. G. Almonte Moreno, licenciado en Psicología por la Universidad de Sevilla y egresado del Máster en Educación y Nuevas Tecnologías de la Universidad a Distancia de Madrid (UDIMA).

2 J. Bravo Agapito, profesor de la Universidad a Distancia de Madrid (UDIMA).
} 


\section{Gamification and C-learning: study of a university context for the adaptation of the design}

\begin{abstract}
:
Gamification, applied to educational contexts, can increase the motivation and engagement of students. First analysis of the bibliography tells that there is not enough research done in this topic, and there are few guidelines marked to implement gamification. This work aims to generate hypotheses that guide the design of a future pilot study in e-learning high education. Students and teachers from the Master of Education and New Technologies at the Madrid Open University (UDIMA) have been tested. The methodology includes qualitative and quantitative aspects and two different on-line questionnaires. Student's questionnaire included a translation of the BrainHex test. Conclusions about the gamification characteristics which are more suitable in this e-learning high education context are generated using these results and through a review of existing research: related to the acceptance of gamification by students and teachers; related to the type of gamification elements that are more suitable for use in the context at the UDIMA and related to aspects or behaviors that need to be changed through the motivation of students.
\end{abstract}

Keywords: gamification, motivation, engagement, e-learning, BrainHex model, higher education.

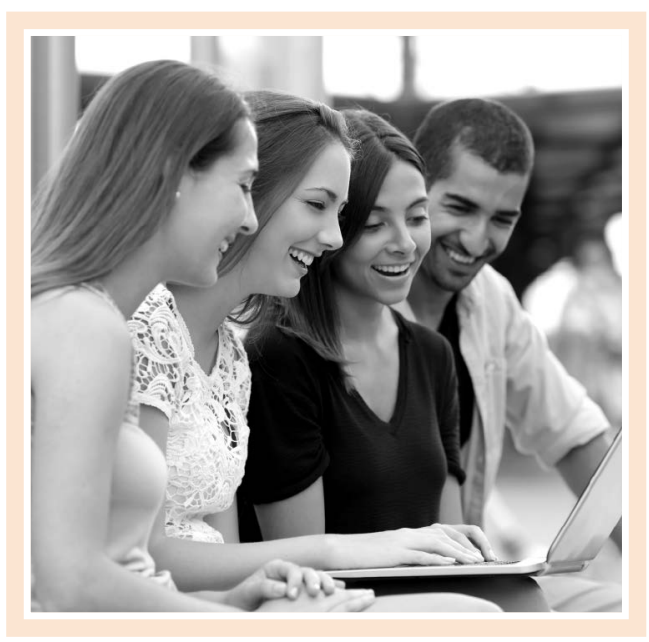

\section{INTRODUCCIÓN Y OBJETIVOS}

La gamificación (procedente del neologismo en inglés gamification) es un concepto relacionado con el marketing, la motivación y la fidelización de los usuarios (Cortizo et ál., 2011). Básicamente, consiste en introducir elementos y vivencias propias de los juegos en contextos no lúdicos, como es, en este caso, el ámbito educativo.

A diferencia de los "juegos educativos» o los serious games, la gamificación puede dirigirse a objetivos más concretos, como son motivar al alumnado, mejorar su implicación, su productividad o su nivel de satisfacción. Todo ello, mediante la introducción de elementos de diseño como puntos, recolección, clasificaciones, niveles, una historia de fondo, actividades creativas o feedback, $u$ otros cambios que recreen experiencias similares a las vividas en los juegos.

Diseñar e implementar de forma adecuada estas técnicas de gamificación en la oferta formativa puede suponer ventajas para el alumnado, para el profesorado y para la institución, aumentando la motivación, el grado de compromiso, automatizando procesos y haciendo más atractiva dicha oferta formativa, entre otras. 
La investigación sobre gamificación puede constituir un reto para la UDIMA, en tanto en cuanto podría devenir, por un lado, en una mejora educativa $y$, por otro, en una valiosa aportación a la comunidad científica internacional, ofreciendo datos acerca de cómo es de efectivo, en términos pedagógicos, el uso de esta tecnología.

El objetivo general del presente trabajo es generar hipótesis acerca de qué tipos de elementos de gamificación son más apropiados para utilizar y a la motivación de qué conductas 0 actitudes deberían dirigirse a la hora de diseñar un curso o asignatura para poner en práctica con los alumnos matriculados en el Máster en Educación y Nuevas Tecnologías impartido por la UDIMA.

Como objetivos específicos se establecen los siguientes:

- Evaluar las preferencias del alumnado actualmente matriculado en el Máster de Educación y Nuevas Tecnologías de la UDIMA hacia elementos relacionados con los juegos.

- Evaluar el conocimiento del profesorado acerca de la gamificación. Conocer su opinión sobre su implantación en el ámbito universitario de la formación a distancia, así como en relación a las áreas en las que podría ser susceptible incidir con un diseño gamificado.

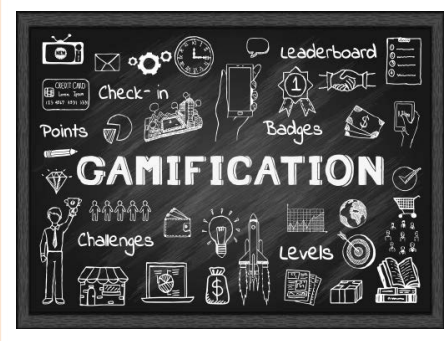
A diferencia de los «juegos educativos» 0 los serious games, la gamificación puede dirigirse a objetivos más concretos, como son motivar al alumnado, mejorar su implicación, su productividad o su nivel de satisfacción. Todo ello, mediante la introducción de elementos de diseño como puntos, recolección, clasificaciones, niveles, una historia de fondo, actividades creativas o feedback, u otros cambios que recreen experiencias similares a las vividas en los juegos

\section{TRABAJOS RELACIONADOS}

\subsection{Gamificación, aprendizaje y e-learning}

Glover (2013) describe la correlación existente entre el diseño de juegos y el de situaciones de aprendizaje a través de tres conceptos claves de gamificación que son aplicables a la educación:

- Actividad enfocada hacia objetivos.

- Mecanismos de recompensa.

- Seguimiento del progreso.

Según refiere este autor, la gamificación es un proceso que, aplicado a la educación, puede crear experiencias participativas de aprendizaje y está especialmente indicado para el aprendizaje activo.

En relación a los elementos de gamificación que pueden ser aplicados en el ámbito educativo, destacamos la revisión de diferentes estudios de casos realizada por Nah et ál. (2014), enumerando mecánicas y componentes de diseño de gamificación que han sido usados por diferentes autores, así como los posibles resultados de su aplicación. Entre ellos pueden destacarse elementos como puntos, niveles, insignias, retos, reintentos, elementos visuales y recompensas dentro del juego. 


\subsection{Evaluación del alumnado para la adapta- ción de un diseño gamificado}

Dado que la gamificación hace referencia a experiencias relacionadas con el juego, cabe pensar que conocer las características personales de los estudiantes y sus preferencias en torno a este tema puede ofrecer indicadores acerca de qué tipo de elementos y mecánicas de juego podrían tener mayor garantía de éxito y adaptarse mejor a sus necesidades.

En la aplicación de la gamificación a distintos ámbitos, incluido el del aprendizaje, diversos autores -como Ferro, Walz y Greuter (2013), Glover (2013), Muntean (2011), O'Donovan, Gain y Marais (2012) u Ong et ál. (2013)-, destacan la importancia de tener en cuenta la personalidad de los usuarios y/o su tipología de jugadores en el diseño. En este sentido, para evaluar la satisfacción con las diferentes experiencias de juego, existen una variedad de modelos que clasifican y describen distintas tipologías de jugadores, y que han sido ampliamente utilizados, principalmente para adaptar el diseño de videojuegos (Nacke, Bateman y Mandryk, 2013).

Este tipo de clasificación también ofrece información relacionada con la personalidad del individuo, existiendo una propuesta teórica (Ferro et ál., 2013) que relaciona tipos de jugadores y tipos de personalidad con elementos y mecánicas de juego, y que constituyen un marco de interpretación de los datos que se han obtenido durante este estudio. A continuación se señalan los modelos más relevantes:

\section{Diseñar e implementar de forma adecuada estas técnicas de gamificación en la oferta formativa puede suponer ventajas para el alumnado, para el profesorado y para la institución, aumentando la motivación, el grado de compromiso, automatizando procesos y haciendo más atractiva dicha oferta formativa, entre otras}

- Test de Bartle. Establece cuatro categorías diferentes (Bartle, 1996):

- Achiever (jugador que considera la recolección de puntos como su principal motivación en el juego).

- Socialiser (más interesado en las interacciones sociales que el juego proporciona que en las mecánicas en sí mismas).

- Explorer (más interesado en descubrir el funcionamiento del mundo del juego; le gusta poner a prueba las mecánicas).

- Killer (jugador muy competitivo, interesado en imponerse sobre los demás).

- Modelo y test de BrainHex. Propuesto por Nacke, Bateman y Mandryck (2013), utiliza siete tipos diferentes:

- Seeker (motivado por la curiosidad, disfruta los momentos de inmersión sensorial).

- Survivor (disfruta con la activación que le producen las situaciones de terror y la intensidad de la experiencia asociada).

- Daredevil (disfruta principalmente de la emoción que proporcionan experiencias de riesgo y velocidad).

- Mastermind (amante de las situaciones que requieren la toma de decisiones estratégicas o la solución de rompecabezas).

- Conqueror (disfrutan luchando contra la adversidad, contra enemigos imposibles u otros jugadores).

- Socialiser.

- Achiever.

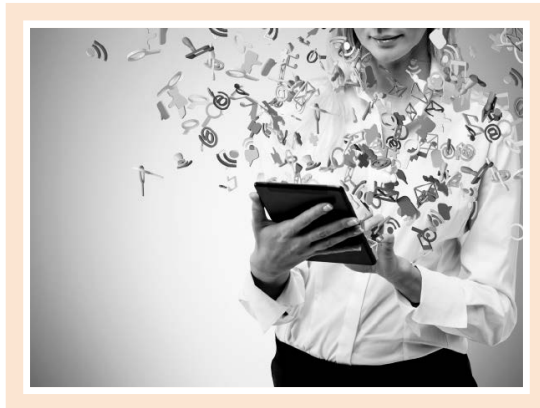


- Modelo de Andrzej Marczewski. Se trata del modelo de más reciente creación (2013) y está dirigido específicamente a valorar qué elementos de gamificación son más apropiados para los usuarios, enmarcándolos en $6+2$ posibles categorías:

- Networkers (buscan contactos y siguen a gente influyente con la esperanza de que estos los recompensen).

- Exploiters (exploran los límites del sistema para usarlo en su beneficio).

- Consumers (hacen lo que haga falta para obtener recompensas, sin importarles que haya o no un esfuerzo o emoción especial asociada a ello).

- Self seekers (responden preguntas de la gente y comparten conocimiento, pero siempre a cambio de una recompensa).

- Socialisers (motivados por las relaciones sociales).

- Free spirits (son amantes de la libertad en el juego, ya sea para explorar o bien para crear).

- Achievers (interesados en conseguir el $100 \%$ de los logros mediante el conocimiento de todas las mecánicas).

- Philanthropists (les gusta sentir que están ayudando a los demás).

La gamificación (procedente del neologismo en inglés gamification) es un concepto relacionado con el marketing, la motivación y la fidelización de los usuarios (Cortizo et ál., 2011). Básicamente, consiste en introducir elementos y vivencias propias de los juegos en contextos no lúdicos, como es, en este caso, el ámbito educativo

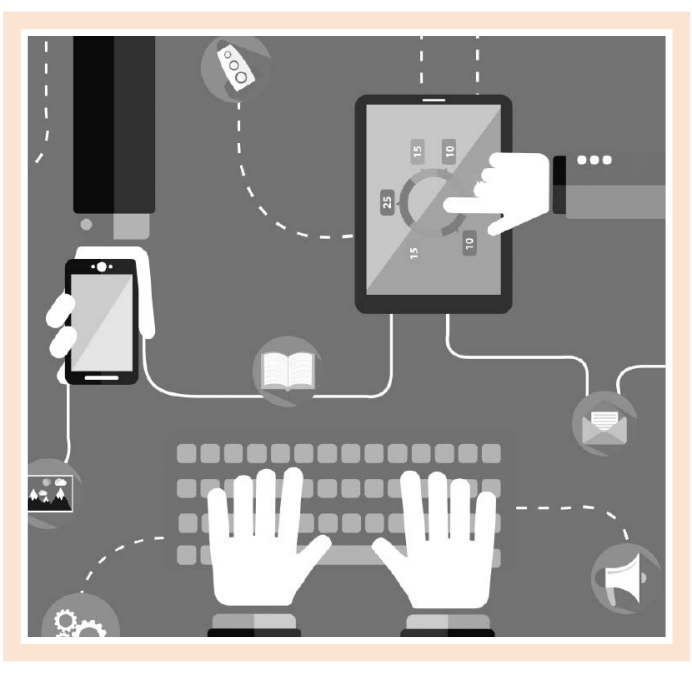

\section{METODOLOGÍA}

\subsection{Diseño de la investigación}

Para realizar el diseño de esta investigación se ha tenido en cuenta que el fin de este trabajo es generar hipótesis que puedan ser útiles para poner en marcha una experiencia real de gamificación y/o un estudio piloto. Debido a ello se ha combinado la metodología cualitativa y cuantitativa con una finalidad descriptiva y exploratoria, aplicada a un contexto concreto.

Para evaluar el contexto universitario de la UDIMA, el diseño de esta investigación tiene en cuenta dos agentes importantes:

- El alumnado, en tanto en cuanto debe adaptarse el diseño gamificado a sus características.

- El profesorado, ya que será esta figura la que incorporará la gamificación a la práctica educativa, concretando su utilización en el proceso formativo.

En base a lo anterior, el diseño de investigación se concreta en tres pasos:

- Recogida de datos del alumnado y el profesorado.

- Análisis de datos.

- Interpretación de los resultados y generación de propuestas a la luz del marco teórico y experiencias documentadas. 


\subsection{Recogida y análisis de datos}

Para la recogida de datos se enviaron cuestionarios al alumnado del Máster de Educación y Nuevas Tecnologías entre los meses de mayo y junio de 2014 y a su profesorado en el mismo periodo utilizando la herramienta de cuestionarios que proporciona Google Drive. La muestra utilizada estuvo formada por 31 alumnos y 8 profesores.

\subsubsection{Cuestionario del alumnado}

El cuestionario dirigido al alumnado incluyó ítems destinados a recoger datos sobre las siguientes áreas:

- Datos sociodemográficos. Se recogieron datos sobre la edad, el género y la frecuencia en el uso de videojuegos por parte de los alumnos participantes.

- Motivación del alumnado hacia distintos tipos de elementos gamificados. El alumnado realizó una valoración acerca de cómo creía que influiría en su motivación la presencia de ciertos elementos que se utilizan habitualmente en diseños instruccionales gamificados. Cada uno de los elementos propuestos debía ser valorado en una escala Likert de 5 valores, desde «bastante desmotivador» hasta «bastante motivador».

- Tipos de jugadores según el modelo BrainHex. Teniendo en cuenta la definición de gamificación aportada por Werbach (2014), en primer lugar, se decidió evaluar cuáles eran los tipos de experiencias de juego que prefiere el alumnado matriculado en el Máster en Educación y Nuevas Tecnologías, con el fin de recabar datos cuantitativos y cualitativos que ayudaran a generar una reflexión acerca de qué estrategias y elementos de juego pudieran ser más adecuados de usar para ajustarse a las características de esta población.

Para ello, se incluyó en el cuestionario una traducción de elaboración propia del cuestionario BrainHex (Bateman, 2010), basado en el modelo de Bartle (1996), que tiene como fin determinar el tipo de experiencia de juego que prefieren los participantes.

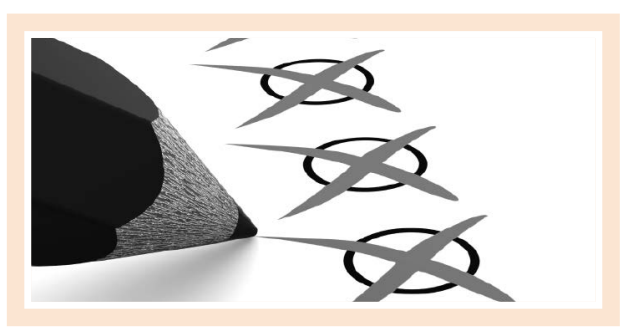

\subsubsection{Cuestionario del profesorado}

El cuestionario dirigido al profesorado del Máster en Educación y Nuevas Tecnologías tenía como objetivos, en primer lugar, describir la situación de este colectivo acerca de su conocimiento y uso de técnicas de gamificación, así como su opinión sobre su implantación en el ámbito universitario de la formación a distancia. En segundo lugar, trató de recabar su punto de vista en relación a qué indicadores o conductas sería necesario promover mediante la motivación del alumnado.

El cuestionario, de elaboración propia, constaba de tres áreas de exploración:

- Conocimiento y uso de técnicas de gamificación.

- Opinión del profesorado respecto a la aplicación de gamificación.

- Necesidades percibidas por el profesorado respecto a la motivación del alumnado.

La investigación sobre gamificación puede constituir un reto para la UDIMA, en tanto en cuanto podría devenir, por un lado, en una mejora educativa y, por otro, en una valiosa aportación a la comunidad científica internacional, ofreciendo datos acerca de cómo es de efectivo, en términos pedagógicos, el uso de esta tecnología 


\section{RESULTADOS}

\subsection{Resultados del cuestionario del alumnado}

\subsubsection{Datos sociodemográficos}

La edad media de los participantes fue de 33 años. El estudiante más joven contaba con 22 años y el mayor con 53. La muestra en su mayor parte fueron mujeres, con un total de $20(64,5 \%)$, frente a 11 hombres (35,5\%). La mayor parte del alumnado manifestó utilizar videojuegos rara u ocasionalmente.

\subsubsection{Motivación del alumnado hacia distintos tipos de elementos gamificados}

Todos los ítems (correspondientes a elementos que se utilizan habitualmente en diseños instruccionales gamificados) fueron valorados en su mayoría como algo o bastante motivadores, si bien las «tablas de clasificaciones» y los «contenidos desbloqueables» obtuvieron más respuestas neutras o negativas (consideración de no influencia o de ser algo desmotivador).

\subsubsection{Tipos de jugadores según el modelo BrainHex}

El análisis de los resultados obtenidos reveló que teniendo en cuenta el modelo BrainHex, los alumnos estudiados están fuertemente orientados en el juego hacia la consecución de metas a largo plazo, obtienen satisfacción completando tareas y coleccionando cosas (achiever). Asimismo, les gusta resolver acertijos y planificar, así como elaborar estrategias eficientes (mastermind).

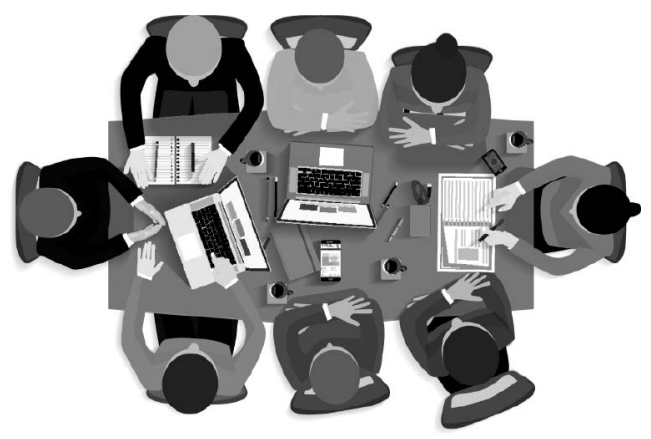

Otorgar al alumnado un papel protagonista en la fase de diseño de gamificación puede redundar en un diseño gamificado más significativo y, por lo tanto, favorecer su implicación y motivación en el proceso de aprendizaje

\subsection{Resultados del cuestionario del profesorado}

De los datos obtenidos podemos destacar aquellos relacionados con su opinión acerca de cuáles son los indicadores o conductas del alumnado en las que se podría incidir mejorando su motivación y en qué grado consideran que sería necesario actuar para cada una de ellas -en términos de beneficio tanto para el proceso de aprendizaje del estudiantado como para la dinámica de la institución que imparta la docencia-.

Los indicadores claramente percibidos como más necesarios se pueden resumir en los siguientes:

- «Aumentar la participación en los foros para aportar recursos, comentarios u opiniones sobre la temática de la asignatura».

- «Entregar las actividades de aprendizaje o evaluación continua con mayor antelación».

- «Aumentar la calidad del trabajo realizado (grado de esfuerzo) en las actividades de aprendizaje o evaluación continua individuales». 


\section{CONCLUSIONES}

A continuación se presentan los factores que, según los resultados obtenidos y la revisión de trabajos relacionados, deberían ser tenidos en cuenta para diseñar un estudio piloto en el contexto del Máster Universitario de Educación y Nuevas Tecnologías de la UDIMA:

- La introducción de gamificación en el aula virtual tendrá un buen nivel de aceptación entre el alumnado del Máster Universitario en Educación y Nuevas tecnologías de la UDIMA.

- Con el fin de mejorar la motivación y la participación, puede considerarse adecuado incluir a los alumnos los siguientes elementos de gamificación: insignias, bonificaciones, combos, niveles, barra de progreso, recompensas programadas, inventario, personalización, misiones o retos y estatus visible (privado).

- También podría ser efectiva la aplicación de los siguientes elementos: puntos de experiencia, feedback inmediato y onboarding (indicaciones paso a paso sobre la interfaz).

- Debería evitarse o moderarse el uso de los siguientes elementos: tabla de clasificaciones, estatus público y contenidos desbloqueables (no previstos por el alumnado).

- Para evitar la reducción de la motivación interna del alumnado, la gamificación debe proporcionar actividades y experiencias diversas que resulten significativas, evitando centrarse en la introducción discreta de elementos basados en motivadores extrínsecos como puntos o recompensas.

- Otorgar al alumnado un papel protagonista en la fase de diseño de gamificación puede redundar en un diseño gamificado más significativo y, por lo tanto, favorecer su implicación y motivación en el proceso de aprendizaje.

- El profesorado muestra una actitud positiva hacia la gamificación, así como expectativas favorables respecto a los beneficios educativos que puede tener la puesta en marcha de un estudio piloto en la UDIMA sobre la implantación de un diseño de gamificación; si bien, para el éxito de esta, el docente deberá colaborar de manera voluntaria, recibir un asesoramiento previo e implicarse activamente.

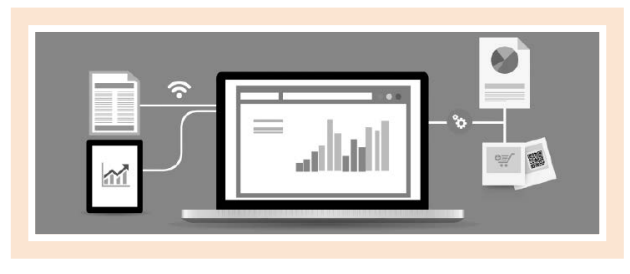

- Debe evitarse explícitamente el uso de elementos de gamificación como única solución a la baja participación y esfuerzo del alumnado en actividades académicas.

- Un estudio piloto en la UDIMA debería iniciarse con objetivos medibles dirigidos hacia áreas con necesidades, pero menos problemáticas, como son la frecuencia de visitas a la plataforma, la frecuencia de realización de actividades optativas, la frecuencia de consulta de recursos y bibliografía complementaria y disminuir la necesidad de plantear dudas técnicas.

- Algunas mecánicas que consideramos de interés para cubrir estos objetivos, de acuerdo a los trabajos relacionados consultados, son insignias, puntos de experiencia y estatus visible (privado), que incentiven la realización de conductas; barra de progreso, para mantener una visión clara del avance; feedback inmediato, que refuerce los logros conseguidos, y onboarding (indicaciones y feedback sobre el uso de la interfaz), que incentive y ayude en el uso técnico de la plataforma on-line.

Este trabajo puede inspirar y guiar la puesta en marcha de estudios de casos u otras investigaciones de corte cuasiexperimental que contribuyan a que la comunidad científica profundice en el concepto de «gamificación» aplicado al aprendizaje y, más en concreto, al ámbito de la formación universitaria en modalidad e-learning o b-learning.

Con el fin de realizar un estudio integral, futuros trabajos deberían incidir también en aspectos institucionales, presupuestarios, estructurales y técnicos de la plataforma de teleformación.

Por último, en cuanto a la evaluación del alumnado, nuevas investigaciones pueden verse beneficiadas en la fiabilidad de sus conclusiones si realizan una doble evaluación de contraste, utilizando, por el ejemplo, el test de Marczewski (2013), realizado específicamente para la gamificación. 


\section{REFERENCIAS BIBLIOGRÁFICAS}

Bartle, R. [1996]: «Hearts, clubs, diamonds, spades: players who suit MUDs», Journal of MUD Research, 1 (1), pág. 19.

Bateman, C. [2010]: BrainHex. What's your brain class? Disponible en: http://blog.brainhex.com/. [Consulta: 23 de marzo de 2014].

Cortizo, J. C.; Carrero, F. M.; Monsalve, B.; Velasco, A.; Díaz del Dedo, L. I. y Pérez, J. [julio 2011]: «Gamificación y docencia: lo que la universidad tiene que aprender de los videojuegos», VIII Jornadas Internacionales de Innovación Universitaria: Retos y Oportunidades del Desarrollo de los Nuevos Títulos en Educación Superior, Madrid (paper).

Ferro, L. S.; Walz, S. P. y Greuter, S. [2013]: «Towards personalised, gamified systems: an investigation into game design, personality and player typologies», Proceedings of The 9th Australasian Conference on Interactive Entertainment: Matters of Life and Death, 7, ACM.

Glover, I. [2013]: «Play as you learn: gamification as a technique for motivating learners», Proceedings of World Conference on Educational Multimedia, Hypermedia and Telecommunications 2013, AACE, Chesapeake (VA), 1999-2008. ISBN: 9781939797032.

Marczewski, A. [2013]: Marczewski's user types hexad. Andrzej's blog. Disponible en: http://www.gamified. co.uk/user-types/ [Consulta: 19 de junio de 2014].

Muntean, C. I. [2011]: «Raising engagement in e-learning through gamification», Proceedings 6th International Conference on Virtual Learning ICVL, págs. 323-329.
Nacke, L. E.; Bateman, C. y Mandryk, R. L. [2013]: «BrainHex: a neurobiological gamer typology survey», Entertainment Computing, 5 (2014), págs. 55-62.

Nah, F. F. H.; Zeng, Q.; Telaprolu, V. R.; Ayyappa, A. P. y Eschenbrenner, B. [2014]: «Gamification of education: a review of literature», $\mathrm{HCl}$ in Business: First International Conference, HCIB 2014, Held as Part of $\mathrm{HCl}$ International 2014, Heraklion, Crete, Greece, June 22-27 2014, Proceedings, Springer International Publishing, págs. 401-409.

O'Donovan, S. [2012]: Gamification of the games course, Technical Report CS12-04-00, Department of Computer Science, University of Cape Town.

O'Donovan, S.; Gain, J. y Marais, P. [2013]: «A case study in the gamification of a university-level games development course», Proceedings of the South African Institute for Computer Scientists and Information Technologists Conference, págs. 242-251.

Ong, D. T.; Chan, Y. Y.; Cho, W. y Koh, T. Y. [2013]: «Motivation of learning: an assessment of the practicality and effectiveness of gamification within a tertiary education system in Malaysia», World Academy of Researchers, Educators, and Scholars in Business, Social Sciences, Humanities and Education Conference, 22-25 July 2013, Cape Town, South Africa.

Werbach, K. [2014]: «(Re)defining gamification», Lecture notes in computer science, vol. 8.462, págs. 266-272. 